Mots. Les langages du politique

$120 \mid 2019$

Nom d'un parti ! Pour une onomastique partisane

\title{
Noms de partis politiques : une onomastique atypique
}

The names of political parties: atypical onomastics

Los nombres de los partidos políticos: una onomástica atípica

Paul Bacot et Michelle Lecolle

\section{OpenEdition}

Journals

Édition électronique

URL : https://journals.openedition.org/mots/25099

DOI : $10.4000 /$ mots.25099

ISSN : 1960-6001

Éditeur

ENS Éditions

Édition imprimée

Date de publication : 11 juillet 2019

Pagination : $9-13$

ISBN : 979-10-362-0170-7

ISSN : 0243-6450

\section{Référence électronique}

Paul Bacot et Michelle Lecolle, "Noms de partis politiques : une onomastique atypique », Mots. Les langages du politique [En ligne], 120 | 2019, mis en ligne le 01 janvier 2021, consulté le 23 avril 2022 URL : http://journals.openedition.org/mots/25099; DOI : https://doi.org/10.4000/mots.25099

\section{(C) ENS Éditions}




\section{Noms de partis politiques : une onomastique atypique}

Les organisations politiques ont des noms. Chacune a le sien, avec parfois des variantes. Cette propriété classe les noms de partis parmi les noms propres. Mais ce sont des noms propres dotés de caractéristiques particulières : ils dénomment des groupes, constitués en tant que tels; ces noms sont choisis par ceux-là mêmes qu'ils vont dénommer collectivement; ils sont formés de mots appartenant au lexique courant et porteurs de sens - à ce titre, le choix du nom d'un parti est un enjeu sémantique (le nom est supposé refléter quelque chose), mais aussi stratégique sur la scène politique (Offerlé, 2012 [1987]).

Le baptême d'un parti est un acte de langage qui le fait exister en tant que tel, et le nom remplit dès lors plusieurs fonctions. Vis-à-vis des adhérents, des militants, voire des sympathisants, c'est le nom d'un collectif auquel des individus adhèrent et dans lequel ils sont appelés à se reconnaitre. Vis-à-vis du corps électoral, et plus largement de l'opinion publique, c'est une marque, mise sur le marché électoral sous la forme de candidats et de programmes. Sur la scène politique d'un pays, il participe du positionnement parmi l'ensemble des acteurs collectifs de même statut : choisir un nom pour un parti suppose de se situer vis-à-vis des autres, coprésents dans le même espace politique, ou ailleurs, mais avec une même couleur politique, ou encore dans une histoire, parfois une filiation. De même que le parti prend position par rapport à d'autres partis, le nom lui-même appartient à un univers, politique bien sûr, mais aussi culturel et historique, un univers peuplé d'autres noms auxquels il fait parfois explicitement écho. Et si, parfois, le parti change de nom, entendant ainsi manifester les changements du parti lui-même, ce changement intervient dans un environnement fait de références diverses, de rapports de force internes et externes et de stratégies.

Le nom de parti peut être caractérisé comme un «nom propre collectif» (Lecolle, 2014), même si, pour le français du moins, sa forme, souvent polylexicale, l'éloigne des noms propres «prototypiques» (dans les termes

Université de Lyon, Sciences Po Lyon, Triangle (CNRS, UMR 5206)

paul.bacot@sciencespo-lyon.fr

Université de Lorraine, CREM

michelle.lecolle@univ-lorraine.fr 
de Jonasson, 1994 ; voir aussi Leroy, 2004), c'est-à-dire des anthroponymes et toponymes, en général monolexicaux et dont le sens est arbitraire - ou devenu arbitraire avec le temps (pour une discussion, voir notamment Coates, 2006 ; Gardiner, 2005 [1953]). En tant que nom collectif, il renvoie tout à la fois à une pluralité interne (les membres du groupe) et au groupe constitué (Lecolle, 2014 ; Lammert, Lecolle, 2014 ; Bonnet dans ce dossier). Cette dualité sémantique - qu'on trouve avec des noms au singulier morphologique (Parti/Union/ Front, etc.) mais aussi au pluriel (Les Républicains, Les Verts, The Black Panthers) - a plusieurs contreparties sémantico-référentielles, et en particulier le fait que, sous un même nom, le groupe et les individus le constituant ont une certaine indépendance; et c'est bien ce qui intervient lorsque le parti (le groupe) disparait, par exemple s'il est dissous (les individus membres ne disparaissent pas pour autant); c'est aussi ce qui est en jeu lorsque, sous un même nom, un parti se vide de ses membres, en acquiert d'autres, bref est changé en quelque sorte de l'intérieur (voir Courtois dans ce dossier à propos des stratégies de partis politiques adverses au Burundi). En outre, le groupe constitué peut être composite, partagé en courants, et ceci a des conséquences diverses quant au maintien ou au changement du nom (voir Treille dans ce dossier à propos du Parti socialiste). Ainsi, le nom propre collectif manifeste un rapport au réel particulièrement complexe, tout à la fois intrinsèquement humain et symbolique, ce qui apparait particulièrement lorsqu'un nom est frappé d'interdit, comme les mots kurde ou Kurdistan dans les noms de partis en Turquie (Akin dans ce dossier).

On l'a dit, le nom de parti est formé de matériau linguistique préexistant, parfois aussi de noms propres. De ce fait, les noms de partis ne surgissent pas ex nihilo, ni sur le plan du lexique employé ni sur celui de la structure syntaxique. Même si, en France du moins, la forme tend actuellement à se renouveler (voir les formes En Marche!, Génération.s. ou, plus installée, la structure [Les + adjectif nominalisé] comme dans Les Républicains, voir Lecolle, 2016 et Bacot, 2018), beaucoup de noms de partis relèvent de "patrons » assez classiques, déjà décrits par Maurice Tournier (1981) et Paul Bacot (2010, 2018), comportant à l'initiale un nom classificatoire tel que parti, front, union, ligue ou force, complété d'éléments (adjectifs, compléments de noms) de choix plus libre et donc signifiant. Cette structure syntaxique classique se retrouve pour d'autres pays (voir, chez Courtois, le cas des noms en français au Burundi) et d'autres langues que le français : en turc, en arabe (voir dans ce dossier Akin, ainsi que Yankaya, Steuer, Zouaoui) ; en anglais, en russe (voir Bonnet et Colas dans ce dossier). Comme le montre le cas du Parti communiste, le nom de parti lui-même peut se transposer dans plusieurs langues et pays, lorsqu'il y correspond à une réalité ou à un projet. C'est sur la base de ces patrons que les noms se déclinent en différentes nuances (descriptives, évocatrices, parlant à la raison, à la mémoire ou aux affects), et sur ces bases aussi que l'imagination peut 
être sollicitée, comme l'illustre la pratique parodique «Toi aussi invente ton groupuscule d'extrême-gauche/d'extrême-droite ", que propose un générateur automatique de noms de groupuscules (voir Krieg-Planque dans ce dossier).

Associé à la structure syntaxique, le lexique employé fait des noms de partis des formes motivées : le nom de parti reflèterait quelque chose... mais la chose en question, de même que la nature de ce reflet sont loin d'être simples. C'est ce que montre le cas, peut-être extrême, très élaboré en tout cas, de En Marche!, qui relève d'une stratégie signifiante où rien n'est laissé au hasard (voir Fretel dans ce dossier), ni la forme injonctive (avec son point d'exclamation), ni les mots, ni les initiales.

Tous les exemples (ou presque) de noms de partis de ce dossier peuvent être envisagés sous l'angle de la ressource que procure le lexique employé en termes d'évocation, qu'il s'agisse de description, de classification, ou encore de référence à des valeurs guerrières, éthiques ou religieuses : valorisation de la force et du pouvoir voire de la violence (partis burundais, Black Panthers), valorisation du progrès, de principes moraux ou religieux (partis islamistes), du peuple, de la démocratie; qu'il s'agisse d'allusion à d'autres organisations, ou à des camps politiques et à ce qu'ils représentent dans la mémoire collective (gauche, Parti socialiste, Parti communiste, partis d'extrême gauche ou d'extrême droite), ou même de référence parodique à des patrons lexicosyntaxiques devenus répétitifs dans le paysage politique français, comme dans le générateur de noms de partis décrit par Krieg-Planque dans ce dossier; qu’il s'agisse enfin de classification selon le camp politique, le pays ou la région, la couleur de peau (Black Panther Party, White Panther Party) - fût-ce de manière ironique et détournée. $\mathrm{Si}$, dans certains cas a contrario, les dénominations ne tendent pas à la description, c'est notamment parce qu'il pèse des interdits sur le choix des mots (mention du territoire, de la religion par exemple en Turquie) - la description prend alors d'autres chemins -, ou parce que la forme se veut résolument nouvelle (En Marche!). Quant au cas du pluriel (Les Républicains, The Black Panthers), la forme elle-même porte la trace de la pluralité sémantique, en rendant possibles divers effets de sens. Dans tous les cas, le nom de parti tend à être motivé - même si, on le sait, la motivation n'est jamais totale, ni ajustée à tous les aspects du référent (ici, le parti). Quête impossible, comme dans l'ensemble des faits linguistiques.

Le nom propre, en général, a une valeur mémorielle : c'est une ressource pour les locuteurs (voir Jonasson, 1994) et un jalon dans la mémoire collective (voir, notamment, Paveau, 2008, pour les toponymes); c'est le cas, on l'a vu, du nom de parti. Néanmoins, beaucoup de noms connaissent des variations, des réductions et troncations, ou le remplacement d'un élément, comme dans les variations autour du nom Black Panther(s) (Party). Parmi les variations dénominatives généralement stabilisées, la siglaison figure au premier plan, et joue un rôle essentiel dans le discours politique (Bacot, Desmarchelier, Honoré éd., 
2011). Plusieurs cas sont représentés dans ce dossier : pour la France, mais aussi pour le Burundi, ou pour les partis kurdes. De fait, en français du moins, la siglaison semble le plus souvent inévitable, du fait de l'économie linguistique qu'elle représente : dans le discours oral comme dans le discours écrit, le sigle est pratique, même si cette réduction formelle implique nécessairement une réduction sémantique. Mais cette perte de sens, ou de précision, peut présenter des avantages, notamment celui d'une certaine ambigüité. Dès lors, ceux qui choisissent le nom du parti pensent à sa siglaison - ou à ses siglaisons possibles. C'est bien ce qu'on peut voir dans le choix du nom En Marche!, qui a pour sigle les initiales de son fondateur et candidat à la présidentielle $(E M)$, soit une double motivation. Outre la facilité mémorielle et l'économie (au moins apparente) de moyens, les bénéfices sont multiples : «glisser» un nouveau nom « sous » un sigle inchangé, comme en France naguère avec UMP passé de Union pour la majorité présidentielle à Union pour un mouvement populaire; jouer sur la double signification des acronymes (Cap21 / Citoyenneté Action Participation pour le $21^{e}$ siècle), en doublant par là même le pouvoir évocateur de la dénomination; brouiller les références et instaurer une résonance dialogique avec des sigles similaires (sigles des différents partis kurdes; sigles des partis burundais).

Le dossier dans son ensemble est traversé de tous ou plusieurs de ces enjeux - stratégie, référence aux autres, mémoire, régularités et ruptures dans la forme, matériau linguistique du nom et motivation, variation, changement, renouvellement. Les contributeurs appartiennent à différentes disciplines : histoire, sciences du langage, science politique principalement qui, toutes, contribuent conjointement à éclairer cet objet particulier, multifacette. Les huit articles représentent néanmoins une grande diversité : diversité de situations (noms de partis fictifs, présents, passés), de pays, de contextes politiques et d'orientations idéologiques. Selon les cas, les articles proposent des comparaisons entre noms de partis, la description d'évolutions d'un même parti et de son nom en lien avec les acteurs politiques, les doctrines, le contexte, les influences des partis (et des noms) les uns sur les autres, ou l'émergence d'un nom.

\section{Références}

BACOT Paul, 2018, "Onomastique partisane», dans Rencontres avec Michel Offerlé, H. Michel, S. Lévêque, J.-G. Contamin éd., Vulaines-sur-Seine, Éditions du Croquant, p. 293-301.

- 2010, «Développement et diversification d'une onomastique politique », Mots. Les langages du politique, ${ }^{\circ}$ 94, p. 47-56.

Bacot Paul, Desmarchelier Dominique, Honoré Jean-Paul éd., 2011, Sigles et acronymes en politique [numéro thématique], Mots. Les langages du politique, nº 95 . COATES Richard A., 2006, «Properhood », Language, vol. LXXXII, n² 2, p. 356-382. 
GARDInER Alan, 2005 [1953], « [The Theory of Proper Names] Théorie des Noms Propres. Essai polémique», Modèles linguistiques, tome XXVI-1, vol.51, p.55-112.

JONASSON Kerstin, 1994, Le nom propre : constructions et interprétations, Louvain-laNeuve, Duculot.

LAMmert Marie, LeColle Michelle, 2014, "Les noms collectifs en français : une vue d'ensemble», Cahiers de lexicologie, nº105, p. 203-222.

LECOLLE Michelle, 2016, "Dénominations émergentes de groupes sociaux», dans La fabrique des mots français, C. Jacquet-Pfau et J.-F. Sablayrolles éd., Limoges, Lambert-Lucas, p. 327-344.

- 2014, "Dénomination de groupes sociaux : approche sémantique et discursive d'une catégorie de noms propres», dans $4^{e}$ Congrès mondial de linguistique française, F. Neveu, P. Blumenthal, L. Hriba et al. éd., Les Ulis, EDP Sciences, p. 2265-2281, http://dx.doi.org/10.1051/shsconf/20140801063 (consulté le 10 mars 2019).

LeroY Sarah, 2004, Le nom propre en français, Paris, Gap, Ophrys.

OfFERLÉ Michel, 2012 [1987], Les partis politiques, Paris, PUF.

PAVEAU Marie-Anne, 2008, «Le toponyme, désignateur souple et organisateur mémoriel. L'exemple du nom de bataille», Mots. Les langages du politique, n 86, p. 23-35.

TOURNIER Maurice, 1981, «Vers une grammaire des dénominations sociopolitiques au début de la Troisième République (1879-1905)», Mots, n² 2, p.51-72. 